\title{
Notes on power LCM matrices
}

\author{
by \\ Shaofang Hong (Chengdu and Haifa)
}

1. Introduction. Let $S=\left\{x_{1}, \ldots, x_{n}\right\}$ be a set of $n$ distinct positive integers. The matrix having the greatest common divisor $\left(x_{i}, x_{j}\right)$ of $x_{i}$ and $x_{j}$ as its $i, j$-entry is called the greatest common divisor (GCD) matrix, denoted by $\left(\left(x_{i}, x_{j}\right)\right)$. The matrix having the least common multiple $\left[x_{i}, x_{j}\right]$ of $x_{i}$ and $x_{j}$ as its $i, j$-entry is called the least common multiple ( $\left.L C M\right)$ matrix, denoted by $\left(\left[x_{i}, x_{j}\right]\right)$. The set $S$ is said to be factor-closed if it contains every divisor of $x$ for any $x \in S$. H. J. S. Smith [14] showed that the determinant of the GCD matrix $\left(\left(x_{i}, x_{j}\right)\right)$ on a factor-closed set $S$ is the product $\prod_{i=1}^{n} \varphi\left(x_{i}\right)$, where $\varphi$ is Euler's totient function. In [14], Smith also considered the determinant of the LCM matrix $[S]_{n}$ on a factor-closed set $S$. It was shown to be the product $\prod_{i=1}^{n} \varphi\left(x_{i}\right) \pi\left(x_{i}\right)$, where $\pi$ is the multiplicative function which is defined for the prime power $p^{r}$ by $\pi\left(p^{r}\right)=-p$. Smith also gave formulas for more general determinants like $\operatorname{det}\left(\left(x_{i}, x_{j}\right)^{\varepsilon}\right)$ and $\operatorname{det}\left(\left[x_{i}, x_{j}\right]^{\varepsilon}\right)$, where $\varepsilon$ is any exponent. Since then many results (see, for example, [1-13]) concerning GCD matrices and LCM matrices have been published.

The set $S$ is said to be $g c d$-closed if $\left(x_{i}, x_{j}\right) \in S$ for all $1 \leq i, j \leq n$. It is clear that a factor-closed set is gcd-closed but not conversely. In [2], Beslin and Ligh extended Smith's result by showing that the determinant of the GCD matrix $\left(\left(x_{i}, x_{j}\right)\right)$ on a gcd-closed set $S=\left\{x_{1}, \ldots, x_{n}\right\}$ is the product $\prod_{k=1}^{n} \alpha_{k}$, where

$$
\alpha_{k}=\sum_{\substack{d \mid x_{k} \\ d \nmid x_{t}, x_{t}<x_{k}}} \varphi(d) .
$$

In [4], Bourque and Ligh generalized Smith's result on LCM matrices by proving that the determinant of the LCM matrix $\left(\left[x_{i}, x_{j}\right]\right)$ on a gcd-closed set $S=\left\{x_{1}, \ldots, x_{n}\right\}$ is the product $\prod_{k=1}^{n} x_{k}^{2} \beta_{k}$, where

2000 Mathematics Subject Classification: Primary 11C20, 11A25.

Supported partially by an NNSF of China (grant no. 10101015) and the Lady Davis Fellowship at the Technion. 


$$
\beta_{k}=\sum_{\substack{d \mid x_{k} \\ d \nmid x_{t}, x_{t}<x_{k}}} g(d),
$$

with the arithmetical function $g$ defined by $g(m)=m^{-1} \sum_{d \mid m} d \mu(d)$, where $\mu$ is the Möbius function.

In [6], Bourque and Ligh showed that if $S=\left\{x_{1}, \ldots, x_{n}\right\}$ is factor-closed then the GCD matrix $\left(\left(x_{i}, x_{j}\right)\right)$ on $S$ divides the LCM matrix $\left(\left[x_{i}, x_{j}\right]\right)$ on $S$ in the ring $M_{n}(\mathbb{Z})$ of $n \times n$ matrices over the integers (i.e., there is an $n \times n$ matrix $A$ with integer entries such that $\left(\left[x_{i}, x_{j}\right]\right)=A\left(\left(x_{i}, x_{j}\right)\right)=$ $\left.\left(\left(x_{i}, x_{j}\right)\right)(A)^{\mathrm{T}}\right)$. Hong [13] proved that such a factorization theorem on LCM and GCD matrices is no longer true in general. In fact, he showed that if $n \leq 3$, then for any gcd-closed set $S=\left\{x_{1}, \ldots, x_{n}\right\}$, the GCD matrix $\left(\left(x_{i}, x_{j}\right)\right)$ on $S$ divides the LCM matrix $\left(\left[x_{i}, x_{j}\right]\right)$ on $S$ in the ring $M_{n}(\mathbb{Z})$. For $n \geq 4$, there exists a gcd-closed set $S=\left\{x_{1}, \ldots, x_{n}\right\}$ such that the GCD matrix $\left(\left(x_{i}, x_{j}\right)\right)$ on $S$ does not divide the LCM matrix $\left(\left[x_{i}, x_{j}\right]\right)$ on $S$ in the ring $M_{n}(\mathbb{Z})$.

From Beslin and Ligh's result [3], one knows that the GCD matrix $\left(\left(x_{i}, x_{j}\right)\right)$ on any set $S=\left\{x_{1}, \ldots, x_{n}\right\}$ of $n$ distinct positive integers is always nonsingular. However, this is not true for LCM matrices in general [1, Remark 5]. From Smith's result [14], one also knows that the LCM matrix on any factor-closed set is nonsingular. Further, it has been conjectured by Bourque and Ligh [4] that the LCM matrix $\left(\left[x_{i}, x_{j}\right]\right)$ on any gcd-closed set $S=\left\{x_{1}, \ldots, x_{n}\right\}$ is nonsingular. In [9-11], Hong systematically investigated the Bourque-Ligh conjecture. Hong [11] proved that the Bourque-Ligh conjecture is true if $n \leq 7$, but not true if $n \geq 8$. Note also that Hong [10] proved that this conjecture is true for a certain class of gcd-closed sets.

Although it follows from Bourque and Ligh's result [5] that the power GCD matrix $\left(\left(x_{i}, x_{j}\right)^{\varepsilon}\right)$ on any set $S=\left\{x_{1}, \ldots, x_{n}\right\}$ of $n$ distinct positive integers is nonsingular, it is not clear that the power LCM matrix $\left(\left[x_{i}, x_{j}\right]^{\varepsilon}\right)$ on any set $S=\left\{x_{1}, \ldots, x_{n}\right\}$ of $n$ distinct positive integers is also nonsingular, where $\varepsilon \geq 2$ is an integer. For the factor-closed case, one knows by [7] that the answer to this question is affirmative. For the gcd-closed case, Hong [12] gave a conjectural answer to this question as follows.

Conjecture ([12]). Let $\varepsilon$ be a given positive integer. Then there must be a positive integer $k(\varepsilon)$, depending only on $\varepsilon$, such that if $n \leq k(\varepsilon)$, then the power LCM matrix $\left(\left[x_{i}, x_{j}\right]^{\varepsilon}\right)$ on any gcd-closed set $S=\left\{x_{1}, \ldots, x_{n}\right\}$ is nonsingular. But for $n \geq k(\varepsilon)+1$, there exists a gcd-closed set $S=$ $\left\{x_{1}, \ldots, x_{n}\right\}$ such that the power LCM matrix $\left(\left[x_{i}, x_{j}\right]^{\varepsilon}\right)$ on $S$ is singular.

The features of GCD matrices are well known, which is due to the nice structure theorem [3, Theorem 1]. However, the features of LCM matrices are less known which may be due to the fact that the convolution of 
arithmetical functions is not always available. So studying LCM matrices is important. In the present paper, our main interest is still in the nonsingularity of power LCM matrices. We will provide an interesting result related to the above conjecture. For a positive integer $x$, let $\nu(x)$ denote the number of distinct prime factors of $x$. We show that if $\varepsilon$ is a positive integer and $S=\left\{x_{1}, \ldots, x_{n}\right\}$ is a gcd-closed set satisfying $\max _{x \in S}\{\nu(x)\} \leq 2$, then the power LCM matrix $\left(\left[x_{i}, x_{j}\right]^{\varepsilon}\right)$ on $S$ is nonsingular.

The set $S$ is said to be lcm-closed if $\left[x_{i}, x_{j}\right] \in S$ for all $1 \leq i, j \leq n$. For example, $S=\{2,3,6,8,24\}$ is lcm-closed. One can easily check that $x \mid \max \{S\}$ for any $x \in S$ if $S$ is lcm-closed. In the fourth section of this paper, we also show that if $\varepsilon$ is a positive integer and $S=\left\{x_{1}, \ldots, x_{n}\right\}$ is an lcm-closed set satisfying $\max _{x \in S}\{\nu(x)\} \leq 2$, then the power LCM matrix $\left(\left[x_{i}, x_{j}\right]^{\varepsilon}\right)$ on $S$ is nonsingular.

In the final section of this paper, we will raise several conjectures to promote further investigations on GCD and LCM matrices.

2. Reductions of the formula for $\operatorname{det}\left(\left[x_{i}, x_{j}\right]^{\varepsilon}\right)$. For any positive integer $\varepsilon$, let the arithmetical function $\zeta_{\varepsilon}$ be defined for any positive integer $m$ by $\zeta_{\varepsilon}(m)=m^{\varepsilon}$. First one has the following result.

Lemma 2.1. The determinant of the matrix $\left(\left[x_{i}, x_{j}\right]^{\varepsilon}\right)$ defined on a gcdclosed set $S=\left\{x_{1}, \ldots, x_{n}\right\}$ is equal to the product $\prod_{k=1}^{n} x_{k}^{2 \varepsilon} \alpha_{\varepsilon, k}$, where

$$
\alpha_{\varepsilon, k}=\sum_{\substack{d \mid x_{k} \\ d \nmid x_{t}, x_{t}<x_{k}}}\left(\frac{1}{\zeta_{\varepsilon}} * \mu\right)(d) .
$$

Proof. This follows immediately from [12, Theorem 5].

In the rest of this paper, without any loss of generality, we assume that $S=\left\{x_{1}, \ldots, x_{n}\right\}$ satisfies $1 \leq x_{1}<\ldots<x_{n}$. Denote by $|A|$ the cardinality of any finite set $A$. In [11] we gave a reduction of the formula for the determinant of the LCM matrix $\left(\left[x_{i}, x_{j}\right]\right)$ by introducing the concept of the greatest-type divisor. In the following we will give a similar reduction for $\alpha_{\varepsilon, k}$ using ideas similar to those in [11]. One needs a generalization of the principle of cross-classification in [9] to give a preliminary reduction of the formula for $\alpha_{\varepsilon, k}$. For an elegant proof, see [13].

Lemma $2.2([9,13])$. Let $R$ be any given finite set and $f$ any complexvalued function defined on $R$. For a subset $T$ of $R$, set $\bar{T}=R \backslash T$. If $R_{1}, \ldots, R_{m}$ are $m$ given distinct subsets of $R$, then

$$
\sum_{x \in \bigcap_{i=1}^{m} \bar{R}_{i}} f(x)=\sum_{x \in R} f(x)+\sum_{t=1}^{m}(-1)^{t} \sum_{1 \leq i_{1}<\ldots<i_{t} \leq m} \sum_{x \in \bigcap_{j=1}^{t} R_{i_{j}}} f(x) .
$$


LEMmA 2.3. Let $n \geq 1$ be an integer. Then

$$
\sum_{d \mid n}\left(\frac{1}{\zeta_{\varepsilon}} * \mu\right)(d)=n^{-\varepsilon}
$$

Proof. This follows immediately from [12, Lemma 7].

LEMmA 2.4. Let $n$ be an integer. Let $S=\left\{x_{1}, \ldots, x_{n}\right\}$ be a gcd-closed set and $x_{1}<\ldots<x_{n}$. If $\alpha_{\varepsilon, k}$ is defined as in (1), then

$$
\alpha_{\varepsilon, k}=x_{k}^{-\varepsilon}+\sum_{t=1}^{k-1}(-1)^{t} \sum_{1 \leq i_{1}<\ldots<i_{t} \leq k-1}\left(x_{k}, x_{i_{1}}, \ldots, x_{i_{t}}\right)^{-\varepsilon} .
$$

Proof. In Lemma 2.2, let $m=k-1$ and $R=\left\{d \in \mathbb{Z}^{+}: d \mid x_{k}, x_{k} \in S\right\}$. For $1 \leq i \leq k-1$, let $R_{i}=\left\{d \in R: d \mid x_{i}, x_{i} \in S\right\}$. Then $R_{i}=\left\{d \in \mathbb{Z}^{+}\right.$: $\left.d \mid\left(x_{k}, x_{i}\right)\right\}$. By Lemma 2.2,

$$
\begin{aligned}
\alpha_{\varepsilon, k}= & \sum_{d \mid x_{k}}\left(\frac{1}{\zeta_{\varepsilon}} * \mu\right)(d) \\
& +\sum_{t=1}^{k-1}(-1)^{t} \sum_{1 \leq i_{1}<\ldots<i_{t} \leq k-1} \sum_{d \mid\left(x_{k}, x_{i_{1}}, \ldots, x_{i_{t}}\right)}\left(\frac{1}{\zeta_{\varepsilon}} * \mu\right)(d) .
\end{aligned}
$$

By Lemma 2.3, $\sum_{d \mid x_{k}}\left(\frac{1}{\zeta_{\varepsilon}} * \mu\right)(d)=x_{k}^{-\varepsilon}$ and for $1 \leq i_{1}<\ldots<i_{t} \leq$ $k-1(1 \leq t \leq k-1)$,

$$
\sum_{d \mid\left(x_{k}, x_{i_{1}}, \ldots, x_{i_{t}}\right)}\left(\frac{1}{\zeta_{\varepsilon}} * \mu\right)(d)=\left(x_{k}, x_{i_{1}}, \ldots, x_{i_{t}}\right)^{-\varepsilon} .
$$

It then follows from (3) and (4) that (2) holds.

Consequently, we obtain a further reduction of the formula for $\alpha_{\varepsilon, k}$.

Lemma 2.5. Let $S=\left\{x_{1}, \ldots, x_{n}\right\}$ be a gcd-closed set. For $1 \leq k \leq n$, let $I_{k}=\left\{i: 1 \leq i \leq k-1\right.$ and $\left.x_{i} \nmid x_{k}\right\}$ and $J_{k}=\{1, \ldots, k-1\} \backslash I_{k}$. Then

$$
\alpha_{\varepsilon, k}=x_{k}^{-\varepsilon}+\sum_{r=1}^{\left|J_{k}\right|}(-1)^{r} \sum_{\substack{i_{1}<\ldots<i_{r} \\ i_{j} \in J_{k}}}\left(x_{k}, x_{i_{1}}, \ldots, x_{i_{r}}\right)^{-\varepsilon} .
$$

Proof. If $\left|I_{k}\right|=0$, then the assertion follows from Lemma 2.4. In what follows let $\left|I_{k}\right| \geq 1$. Note that for $i \in J_{k}$, one has $x_{i} \mid x_{k}$. Since $S$ is gcd-closed, $x_{1} \mid x_{k}$. Thus, $\left|J_{k}\right| \geq 1$. Note also that $\left|I_{k}\right|+\left|J_{k}\right|=k-1$. By Lemma 2.4,

$$
\alpha_{\varepsilon, k}=x_{k}^{-\varepsilon}+\Delta^{\prime}+\Delta \text {, }
$$

where 


$$
\Delta^{\prime}=\sum_{r=1}^{\left|J_{k}\right|}(-1)^{r} \sum_{\substack{i_{1}<\ldots<i_{r} \\ i_{j} \in J_{k}}}\left(x_{k}, x_{i_{1}}, \ldots, x_{i_{r}}\right)^{-\varepsilon}
$$

and

(7) $\Delta=\sum_{r=1}^{\left|J_{k}\right|} \sum_{\substack{i_{1}<\ldots<i_{r} \\ i_{j} \in J_{k}}} \sum_{s=1}^{\left|I_{k}\right|}(-1)^{r+s} \sum_{\substack{t_{1}<\ldots<t_{s} \\ t_{u} \in I_{k}}}\left(x_{k}, x_{i_{1}}, \ldots, x_{i_{r}}, x_{t_{1}}, \ldots, x_{t_{s}}\right)^{-\varepsilon}$.

For any given $t_{1}<\ldots<t_{s}, t_{u} \in I_{k}(1 \leq u \leq s)$. Since $S$ is gcd-closed it follows that $\left(x_{k}, x_{t_{1}}, \ldots, x_{t_{s}}\right) \in S$. Let $x_{l}=\left(x_{k}, x_{t_{1}}, \ldots, x_{t_{s}}\right)$. Then $x_{l} \mid x_{k}$ and $x_{l} \mid x_{t_{u}}$ for $1 \leq u \leq s$. So, $l \in J_{k}$. Then, by (7),

$$
\begin{aligned}
\Delta & =\sum_{s=1}^{\left|I_{k}\right|} \sum_{\substack{t_{1}<\ldots<t_{s} \\
t_{u} \in I_{k}}} \sum_{r=1}^{\left|J_{k}\right|}(-1)^{r+s} \sum_{\substack{i_{1}<\ldots<i_{r} \\
i_{j} \in J_{k}}}\left(x_{k}, x_{i_{1}}, \ldots, x_{i_{r}}, x_{t_{1}}, \ldots, x_{t_{s}}\right)^{-\varepsilon} \\
= & \sum_{s=1}^{\left|I_{k}\right|} \sum_{t_{1}<\ldots<t_{s}} \sum_{\substack{t_{u} \in I_{k} \\
t_{k} \mid-1}} \sum_{\substack{i_{1}<\ldots<i_{r} \\
i_{j} \in J_{k}, i_{j} \neq l}}\left((-1)^{r+s}\left(x_{k}, x_{i_{1}}, \ldots, x_{i_{r}}, x_{t_{1}}, \ldots, x_{t_{s}}\right)^{-\varepsilon}\right. \\
= & \sum_{s=1} \sum_{\left.(-1)^{r+s+1}\left(x_{k}, x_{i_{1}}, \ldots, x_{i_{r}}, x_{l}, x_{t_{1}}, \ldots, x_{t_{s}}\right)^{-\varepsilon}\right)} \sum_{\substack{I_{1}<\ldots<t_{s} \\
t_{u} \in I_{k}}} \sum_{\substack{J_{k} \mid-1 \\
i_{1}<\ldots<i_{r} \\
i_{j} \in J_{k}, i_{j} \neq l}}\left((-1)^{r+s}\left(x_{i_{1}}, \ldots, x_{i_{r}}, x_{l}\right)^{-\varepsilon}\right. \\
& \left.+(-1)^{r+s+1}\left(x_{i_{1}}, \ldots, x_{i_{r}}, x_{l}\right)^{-\varepsilon}\right) \\
& 0 .
\end{aligned}
$$

It follows from (6) and (8) that (5) holds.

Definition ([11]). Let $T$ be a set of distinct positive integers. For any $a, b \in T$ and $a<b$, we say that $a$ is a greatest-type divisor of $b$ in $T$ if $a \mid b$ and the conditions $a|c, c| b, c<b$, and $c \in T$ imply that $c=a$.

LemMA 2.6. Let $S=\left\{x_{1}, \ldots, x_{n}\right\}$ be a gcd-closed set. For $1 \leq k \leq n$, let $R_{k}=\left\{i: 1 \leq i \leq k-1, x_{i}\right.$ is the greatest-type divisor of $x_{k}$ in $\left.S\right\}$. Then

$$
\alpha_{\varepsilon, k}=x_{k}^{-\varepsilon}+\sum_{r=1}^{\left|R_{k}\right|}(-1)^{r} \sum_{\substack{i_{1}<\ldots<i_{r} \\ i_{j} \in R_{k}}}\left(x_{k}, x_{i_{1}}, \ldots, x_{i_{r}}\right)^{-\varepsilon} .
$$

Proof. For $k \leq 2$, the assertion is clearly true. In what follows let $k \geq 3$. Let $J_{k}=\left\{i: 1 \leq i \leq k-1\right.$ and $\left.x_{i} \mid x_{k}\right\}$. Then $\left|J_{k}\right| \geq 1$. It is clear that 
$R_{k} \subseteq J_{k}$. If $\left|J_{k}\right|=1$, then $J_{k}=\{1\}$. Note that $\left|R_{k}\right| \geq 1$. So one has $R_{k}=\{1\}=J_{k}$. Thus by Lemma 2.5, the result is true. In the following let $\left|J_{k}\right| \geq 2$. Let $L_{k}=J_{k} \backslash R_{k}$. We show that $L_{k} \neq \emptyset$. Assuming otherwise implies that $R_{k}=J_{k}$. But $1 \in J_{k}$. Then $1 \in R_{k}$. From $\left|J_{k}\right| \geq 2$, one deduces that there is an $i \in J_{k}, i \neq 1$, such that $i \in J_{k}=R_{k}$. Since $S$ is gcd-closed, one has $x_{1} \mid x_{i}$. This is impossible since $x_{1}, x_{i}$ cannot both be greatest-type divisors of $x_{k}$ in $S$. Therefore the assertion is true. In a similar way to that in (6), one has, by Lemma 2.5,

$$
\alpha_{\varepsilon, k}=x_{k}^{-\varepsilon}+\bar{\Delta}^{\prime}+\bar{\Delta}
$$

where

$$
\bar{\Delta}^{\prime}=\sum_{r=1}^{\left|R_{k}\right|}(-1)^{r} \sum_{\substack{i_{1}<\ldots<i_{r} \\ i_{j} \in R_{k}}}\left(x_{k}, x_{i_{1}}, \ldots, x_{i_{r}}\right)^{-\varepsilon}
$$

and

$$
\begin{aligned}
& \bar{\Delta}=\sum_{r=0}^{\left|R_{k}\right|} \sum_{\substack{i_{1}<\ldots<i_{r} \\
i_{j} \in R_{k}}} \sum_{s=1}^{\left|L_{k}\right|} \sum_{\substack{t_{1}<\ldots<t_{s} \\
t_{u} \in L_{k}}}(-1)^{r+s}\left(x_{k}, x_{i_{1}}, \ldots, x_{i_{r}}, x_{t_{1}}, \ldots, x_{t_{s}}\right)^{-\varepsilon} \\
& =\sum_{s=1}^{\left|L_{k}\right|} \sum_{\substack{\left|R_{k}\right| \\
t_{1}<\ldots<t_{s} \\
t_{u} \in L_{k}}}(-1)^{s} \sum_{r=0} \sum_{\substack{i_{1}<\ldots<i_{r} \\
i_{j} \in R_{k}}}(-1)^{r}\left(x_{k}, x_{i_{1}}, \ldots, x_{i_{r}}, x_{t_{1}}, \ldots, x_{t_{s}}\right)^{-\varepsilon} .
\end{aligned}
$$

To prove the lemma, one needs only to show that $\bar{\Delta}=0$, which we will do in the following.

For any given $t_{1}<\ldots<t_{s}\left(1 \leq s \leq\left|L_{k}\right|\right), t_{u} \in L_{k}, 1 \leq u \leq s$, let $P=\left\{i: i \in R_{k}\right.$, and $x_{t_{u}} \mid x_{i}$ for some $\left.t_{u}, 1 \leq u \leq s\right\}$ and let $Q=R_{k} \backslash P$. Let $|P|=h$ and $|Q|=h^{\prime}$. Clearly, $1 \leq h \leq\left|R_{k}\right|$ and $0 \leq h^{\prime} \leq\left|R_{k}\right|-1$. Then

$$
\begin{aligned}
& \sum_{r=0}^{\left|R_{k}\right|} \sum_{\substack{i_{1}<\ldots<i_{r} \\
i_{j} \in R_{k} \\
h^{\prime}}}(-1)^{r}\left(x_{k}, x_{i_{1}}, \ldots, x_{i_{r}}, x_{t_{1}}, \ldots, x_{t_{s}}\right)^{-\varepsilon} \\
= & \sum_{r^{\prime}=0} \sum_{\substack{i_{1}<\ldots<i_{r^{\prime}} \\
i_{u} \in Q}}^{h} \sum_{r=0}^{h}(-1)^{r+r^{\prime}}\left(x_{k}, x_{i_{1}}, \ldots, x_{i_{r^{\prime}}}, x_{j_{1}}, \ldots, x_{j_{r}}, x_{t_{1}}, \ldots, x_{t_{s}}\right)^{-\varepsilon} \\
& \sum_{\substack{j_{1}<\ldots<j_{r} \\
j_{v} \in P}}^{\sum_{r^{\prime}=0}^{h^{\prime}}} \sum_{\substack{i_{1}<\ldots<i_{r^{\prime}} \\
i_{u} \in Q}} \sum_{\substack{r=0 \\
j_{1}<\ldots<j_{r} \\
j_{v} \in P}}^{h}(-1)^{r+r^{\prime}}\left(x_{k}, x_{i_{1}}, \ldots, x_{i_{r^{\prime}}}, x_{t_{1}}, \ldots, x_{t_{s}}\right)^{-\varepsilon}
\end{aligned}
$$


(since by the definition of $P,\left(x_{j_{1}}, \ldots, x_{j_{r}}, x_{t_{1}}, \ldots, x_{t_{s}}\right)=\left(x_{t_{1}}, \ldots, x_{t_{s}}\right)$ for any $j_{1}<\ldots<j_{r}, j_{v} \in P$ )

$$
\begin{aligned}
= & \sum_{r^{\prime}=0}^{h^{\prime}} \sum_{\substack{i_{1}<\ldots<i_{r^{\prime}} \\
i_{u} \in Q}}(-1)^{r^{\prime}}\left(x_{k}, x_{i_{1}}, \ldots, x_{i_{r^{\prime}}}, x_{t_{1}}, \ldots, x_{t_{s}}\right)^{-\varepsilon} \\
& \times\left(1+\sum_{r=1}^{h}(-1)^{r} \sum_{\substack{j_{1}<\ldots<j_{r} \\
j_{v} \in P}} 1\right) \\
= & \sum_{r^{\prime}=0}^{h^{\prime}} \sum_{\substack{i_{1}<\ldots<i_{r^{\prime}} \\
i_{u} \in Q}}(-1)^{r^{\prime}}\left(x_{k}, x_{i_{1}}, \ldots, x_{i_{r^{\prime}}}, x_{t_{1}}, \ldots, x_{t_{s}}\right)^{-\varepsilon}\left(1+\sum_{r=1}^{h}(-1)^{r}\left(\begin{array}{c}
h \\
r
\end{array}\right)\right) \\
= & \sum_{r^{\prime}=0}^{h^{\prime}} \sum_{\substack{i_{1}<\ldots<i_{r^{\prime}} \\
i_{u} \in Q}}(-1)^{r^{\prime}}\left(x_{k}, x_{i_{1}}, \ldots, x_{i_{r^{\prime}}}, x_{t_{1}}, \ldots, x_{t_{s}}\right)^{-\varepsilon}(1-1)^{h}=0 .
\end{aligned}
$$

It then follows from (9) and (10) that $\bar{\Delta}=0$.

3. The gcd-closed case. Throughout this section, let $S=\left\{x_{1}, \ldots, x_{n}\right\}$ be gcd-closed. For $1 \leq k \leq n$, let $\alpha_{\varepsilon, k}$ be defined as in (1). It is clear that $\alpha_{\varepsilon, 1}=x_{1}^{-\varepsilon}$. We have the following lemmas.

LEMMA 3.1. For $2 \leq k \leq n$, let $x_{k}=p^{e} q^{h}$, where $p$ and $q$ are distinct primes, $e$ and $h$ are positive integers. Then the set of greatest-type divisors of $x$ in $S$ must have the form $\left\{p^{e_{1}} q^{h_{1}}, \ldots, p^{e_{m}} q^{h_{m}}\right\}$, where $1 \leq m \leq \min \{e, h\}$, $0 \leq e_{1}<\ldots<e_{m} \leq e, h \geq h_{1}>\ldots>h_{m} \geq 0$, and $e_{i}+h_{i} \leq e+h-1$ $(i=1, \ldots, m)$.

Proof. Let $R_{k}$ be the set of greatest-type divisors of $x_{k}$ in $S$ and let $\left|R_{k}\right|=m$. Since $x_{k}=p^{e} q^{h}$, one may let $R_{k}=\left\{p^{e_{1}} q^{h_{1}}, \ldots, p^{e_{m}} q^{h_{m}}\right\}$, where $e_{i}$ and $h_{i}(1 \leq i \leq m)$ are nonnegative integers satisfying $0 \leq e_{i} \leq e$, $0 \leq h_{i} \leq h$ and $e_{i}+h_{i} \leq e+h-1$. We claim that for any $i, j \in\{1, \ldots, m\}$, $i \neq j$, we have $e_{i} \neq e_{j}$. Otherwise, there exist $i, j \in\{1, \ldots, m\}, i \neq j$, such that $e_{i}=e_{j}$. Then $p^{e_{i}} q^{h_{i}} \mid p^{e_{j}} q^{h_{j}}$ or $p^{e_{j}} q^{h_{j}} \mid p^{e_{i}} q^{h_{i}}$. This contradicts the fact that $p^{e_{i}} q^{h_{i}}$ and $p^{e_{j}} q^{h_{j}}$ are greatest-type divisors of $x_{k}$ in $S$. Thus $e_{i} \neq e_{j}$ for any $i, j \in\{1, \ldots, m\}, i \neq j$. Similarly, $h_{i} \neq h_{j}$ for $i, j \in\{1, \ldots, m\}, i \neq j$.

Without loss of generality, one may assume that $0 \leq e_{1}<\ldots<e_{m}$. Since $p^{e_{1}} q^{h_{1}}, \ldots, p^{e_{m}} q^{h_{m}}$ are greatest-type divisors, it follows that for any $i, j \in\{1, \ldots, m\}, i \neq j$, both $p^{e_{i}} q^{h_{i}} \nmid p^{e_{j}} q^{h_{j}}$ and $p^{e_{j}} q^{h_{j}} \nmid p^{e_{i}} q^{h_{i}}$. Therefore for any $i \in\{1, \ldots, m-1\}$, it follows from $e_{i}<e_{i+1}$ and $p^{e_{i}} q^{h_{i}} \nmid p^{e_{i+1}} q^{h_{i+1}}$ that $h_{i}>h_{i+1}$. So $h_{1}>\ldots>h_{m} \geq 0$.

It is clear that $e_{i}+h_{i} \leq e+h$ for $1 \leq i \leq m$. Suppose that there exists $1 \leq i \leq m$ such that $e_{i}+h_{i}=e+h$. One can deduce that $e_{i}=e$ and $h_{i}=h$. 
So $p^{e_{i}} q^{h_{i}}=x_{k}$. This contradicts the fact that $p^{e_{i}} q^{h_{i}}$ is a greatest-type divisor of $x_{k}$. Then $e_{i}+h_{i} \leq e+h-1$ for $i=1, \ldots, m$.

Lemma 3.2. For $2 \leq k \leq n$, let $x_{k}=p^{e} q^{h}$, where $p$ and $q$ are distinct primes, $e$ and $h$ are positive integers. If the set of greatest-type divisors of $x_{k}$ in $S$ is $\left\{p^{e_{1}} q^{h_{1}}, \ldots, p^{e_{m}} q^{h_{m}}\right\}$, where $1 \leq m \leq \min \{e, h\}, 0 \leq e_{1}<\ldots<$ $e_{m} \leq e, h \geq h_{1}>\ldots>h_{m} \geq 0$, and $e_{i}+h_{i} \leq e+h-1(i=1, \ldots, m)$, then $\alpha_{\varepsilon, k}=\left\{\begin{array}{lc}p^{-\varepsilon e} q^{-\varepsilon h}-p^{-\varepsilon e_{1}} q^{-\varepsilon h_{1}} & \text { if } m=1, \\ p^{-\varepsilon e} q^{-\varepsilon h}-p^{-\varepsilon e_{m}} q^{-\varepsilon h_{m}}+\sum_{i=1}^{m-1}\left(p^{-\varepsilon e_{i}} q^{-\varepsilon h_{i+1}}-p^{-\varepsilon e_{i}} q^{-\varepsilon h_{i}}\right) & \text { if } m \geq 2 .\end{array}\right.$

Proof. Let $m \leq 2$. Then by Lemma 2.6, the result is clearly true.

In what follows let $m \geq 3$. Noting that $0 \leq e_{1}<\ldots<e_{m} \leq e$ and $h \geq h_{1}>\ldots>h_{m} \geq 0$, by Lemma 2.6 one has

$$
\begin{aligned}
\alpha_{\varepsilon, k}= & p^{-\varepsilon e} q^{-\varepsilon h}-p^{-\varepsilon e_{1}} q^{-\varepsilon h_{1}}-\ldots-p^{-\varepsilon e_{m}} q^{-\varepsilon h_{m}} \\
& +\sum_{t=2}^{m}(-1)^{t} \sum_{1 \leq i_{1}<\ldots<i_{t} \leq m}\left(p^{e} q^{h}, p^{e_{i_{1}}} q^{h_{i_{1}}}, \ldots, p^{e_{i_{t}}} q^{h_{i_{t}}}\right)^{-\varepsilon} \\
= & p^{-\varepsilon e} q^{-\varepsilon h}-p^{-\varepsilon e_{1}} q^{-\varepsilon h_{1}}-\ldots-p^{-\varepsilon e_{m}} q^{-\varepsilon h_{m}} \\
& +\sum_{t=2}^{m}(-1)^{t} \sum_{1 \leq i_{1}<\ldots<i_{t} \leq m} p^{-\varepsilon e_{i_{1}}} q^{-\varepsilon h_{i_{t}}} \\
= & p^{-\varepsilon e} q^{-\varepsilon h}-p^{-\varepsilon e_{1}} q^{-\varepsilon h_{1}}-\ldots-p^{-\varepsilon e_{m}} q^{-\varepsilon h_{m}} \\
& +p^{-\varepsilon e_{1}} q^{-\varepsilon h_{2}}+\ldots+p^{-\varepsilon e_{m-1}} q^{-\varepsilon h_{m}}+C,
\end{aligned}
$$

where

$$
C=\sum_{t=2}^{m}(-1)^{t} \sum_{\substack{1 \leq i_{1}<\ldots<i_{t} \leq m \\ i_{1}+1<i_{t}}} p^{-\varepsilon e_{i_{1}}} q^{-\varepsilon h_{i_{t}}} .
$$

Since $a+1<b$ implies that $b-a-1 \geq 1$, one has

$$
\begin{aligned}
C & =\sum_{2 \leq a+1<b \leq m} \sum_{t=2}^{b-a+1}(-1)^{t} \sum_{a=i_{1}<\ldots<i_{t}=b} p^{-\varepsilon e_{i_{1}}} q^{-\varepsilon h_{i_{t}}} \\
& =\sum_{2 \leq a+1<b \leq m} \sum_{t=2}^{b-a+1}(-1)^{t} p^{-\varepsilon e_{a}} q^{-\varepsilon h_{b}} \sum_{\substack{a=i_{1}<\ldots<i_{t}=b\\
}} 1 \\
& =\sum_{2 \leq a+1<b \leq m} \sum_{t=2}^{b-a+1}(-1)^{t} p^{-\varepsilon e_{a}} q^{-\varepsilon h_{b}}\left(\begin{array}{c}
b-a-1 \\
t-2
\end{array}\right)
\end{aligned}
$$




$$
\begin{aligned}
& =\sum_{2 \leq a+1<b \leq m} p^{-\varepsilon e_{a}} q^{-\varepsilon h_{b}} \sum_{t=2}^{b-a+1}(-1)^{t-2}\left(\begin{array}{c}
b-a-1 \\
t-2
\end{array}\right) \\
& =\sum_{2 \leq a+1<b \leq m} p^{-\varepsilon e_{a}} q^{-\varepsilon h_{b}} \sum_{l=0}^{b-a-1}(-1)^{l}\left(\begin{array}{c}
b-a-1 \\
l
\end{array}\right) \\
& =\sum_{2 \leq a+1<b \leq m} p^{-\varepsilon e_{a}} q^{-\varepsilon h_{b}}(1-1)^{b-a-1} \\
& =\sum_{2 \leq a+1<b \leq m} p^{-\varepsilon e_{a}} q^{-\varepsilon h_{b}} \cdot 0=0 .
\end{aligned}
$$

Therefore

$$
\begin{aligned}
\alpha_{\varepsilon, k}= & p^{-\varepsilon e} q^{-\varepsilon h}-p^{-\varepsilon e_{1}} q^{-\varepsilon h_{1}}-\ldots-p^{-\varepsilon e_{m}} q^{-\varepsilon h_{m}} \\
& +p^{-\varepsilon e_{1}} q^{-\varepsilon h_{2}}+\ldots+p^{-\varepsilon e_{m-1}} q^{-\varepsilon h_{m}}
\end{aligned}
$$

as desired.

Now we give the first main result in this paper.

TheOREM 3.3. Let $S=\left\{x_{1}, \ldots, x_{n}\right\}$ be a gcd-closed set satisfying $\max _{x \in S}\{v(x)\} \leq 2$ and $\varepsilon$ a positive integer. Then the power LCM matrix $\left(\left[x_{i}, x_{j}\right]^{\varepsilon}\right)$ on $S$ is nonsingular.

Proof. One may assume $x_{1}<\ldots<x_{n}$. Since $\max _{x \in S}\{v(x)\} \leq 2$, for $2 \leq k \leq n$, noting that $x_{k}>1$, one has $v\left(x_{k}\right)=1$ or 2 . Namely, $x_{k}=p^{e}$, where $e \geq 1$ is an integer and $p$ is a prime, or $x_{k}=p^{e} q^{h}$, where $e \geq 1$ and $h \geq 1$ are integers, $p$ and $q$ are distinct primes. We claim that $\alpha_{\varepsilon, k} \neq 0$ for $1 \leq k \leq n$.

If $k=1$, then $\alpha_{\varepsilon, 1}=x_{1}^{-\varepsilon} \neq 0$ by Lemma 2.5, so the claim is true. In the following let $k \geq 2$. Consider the following two cases.

CASE 1: $x_{k}=p^{e}$. Then $x_{k}$ has only one greatest-type divisor in $S$ whose form must be $p^{l}$, where $l$ is an integer and $0 \leq l \leq e-1$. By Lemma 2.5, $\alpha_{\varepsilon, k}=p^{-\varepsilon e}-p^{-\varepsilon l}$. Since $\varepsilon$ is a positive integer, one then deduces that $\alpha_{\varepsilon, k}<0$. The claim is true.

CASE 2: $x_{k}=p^{e} q^{h}$. It follows from Lemma 3.1 that there exist $2 m$ (where $1 \leq m \leq \min \{e, h\}$ ) integers $e_{1}, \ldots, e_{m}, h_{1}, \ldots, h_{m}$ satisfying $0 \leq$ $e_{1}<\ldots<e_{m} \leq e, h \geq h_{1}>\ldots>h_{m} \geq 0$, and $e_{i}+h_{i} \leq e+h-1$ $(i=1, \ldots, m)$, such that $\left\{p^{e_{1}} q^{h_{1}}, \ldots, p^{e_{m}} q^{h_{m}}\right\}$ is equal to the set of greatesttype divisors of $x_{k}$ in $S$. If $m=1$, then $\alpha_{\varepsilon, k}=p^{-\varepsilon e} q^{-\varepsilon h}-p^{-\varepsilon e_{1}} q^{-\varepsilon h_{1}}$ by Lemma 3.2. By the assumption, $\alpha_{\varepsilon, k}<0$, so the claim is true. In the following let $m \geq 2$. By Lemma 3.2, 
(11)

$$
\begin{aligned}
\alpha_{\varepsilon, k}= & \frac{1}{p^{\varepsilon e} q^{\varepsilon h}}-\frac{1}{p^{\varepsilon e_{1}} q^{\varepsilon h_{1}}}-\ldots-\frac{1}{p^{\varepsilon e_{m}} q^{\varepsilon h_{m}}} \\
& +\frac{1}{p^{\varepsilon e_{1}} q^{\varepsilon h_{2}}}+\ldots+\frac{1}{p^{\varepsilon e_{m-1}} q^{\varepsilon h_{m}}} \\
= & \frac{1}{p^{\varepsilon e} q^{\varepsilon h}}+\left(\frac{1}{p^{\varepsilon e_{1}} q^{\varepsilon h_{2}}}-\frac{1}{p^{\varepsilon e_{1}} q^{\varepsilon h_{1}}}-\frac{1}{p^{\varepsilon e_{2}} q^{\varepsilon h_{2}}}\right) \\
& +\left(\frac{1}{p^{\varepsilon e_{2}} q^{\varepsilon h_{3}}}-\frac{1}{p^{\varepsilon e_{3}} q^{\varepsilon h_{3}}}\right)+\ldots+\left(\frac{1}{p^{\varepsilon e_{m-1}} q^{\varepsilon h_{m}}}-\frac{1}{p^{\varepsilon e_{m}} q^{\varepsilon h_{m}}}\right) .
\end{aligned}
$$

Since $e_{1}<e_{2}$ and $h_{1}>h_{2}$, one has $e_{2}-e_{1} \geq 1$ and $h_{1}-h_{2} \geq 1$. Since $\varepsilon \geq 1$, one can deduce that $\left(p^{\varepsilon\left(e_{2}-e_{1}\right)}-1\right)\left(q^{\varepsilon\left(h_{1}-h_{2}\right)}-1\right)-1 \geq(2-1)(3-1)-1$ $=1$. Then

$$
\begin{aligned}
\frac{1}{p^{\varepsilon e_{1}} q^{\varepsilon h_{2}}}-\frac{1}{p^{\varepsilon e_{1}} q^{\varepsilon h_{1}}} & -\frac{1}{p^{\varepsilon e_{2}} q^{\varepsilon h_{2}}} \\
= & \frac{1}{p^{\varepsilon e_{2}} q^{\varepsilon h_{1}}}\left[\left(p^{\varepsilon\left(e_{2}-e_{1}\right)}-1\right)\left(q^{\varepsilon\left(h_{1}-h_{2}\right)}-1\right)-1\right]>0 .
\end{aligned}
$$

For $i=3, \ldots, m$, since $e_{i-1}<e_{i}, e_{i}-e_{i-1} \geq 1$, one has

$$
\frac{1}{p^{\varepsilon e_{i-1}} q^{\varepsilon h_{i}}}-\frac{1}{p^{\varepsilon e_{i}} q^{\varepsilon h_{i}}}=\frac{p^{\varepsilon\left(e_{i}-e_{i-1}\right)}-1}{p^{\varepsilon e_{i}} q^{\varepsilon h_{i}}}>0 \text {. }
$$

By (11)-(13), one has $\alpha_{\varepsilon, k}>0$.

It then follows from the claim and Lemma 2.1 that $\operatorname{det}\left(\left[x_{i}, x_{j}\right]^{\varepsilon}\right) \neq 0$. Therefore the power LCM matrix $\left(\left[x_{i}, x_{j}\right]^{\varepsilon}\right)$ on $S$ is nonsingular.

4. The lcm-closed case. In this section, we transfer the result of Section 3 to the lcm-closed case by using the following lemmas.

LEMMA 4.1. Let $S=\left\{x_{1}, \ldots, x_{n}\right\}$ be a set of $n$ distinct positive integers. Let $\varepsilon$ be a real number and let $m=\operatorname{lcm}\{S\}$. Then

$$
\left(\left[x_{i}, x_{j}\right]^{\varepsilon}\right)=\frac{1}{m^{\varepsilon}} \cdot \operatorname{diag}\left(x_{1}^{\varepsilon}, \ldots, x_{n}^{\varepsilon}\right) \cdot\left(\left[\frac{m}{x_{i}}, \frac{m}{x_{j}}\right]^{\varepsilon}\right) \cdot \operatorname{diag}\left(x_{1}^{\varepsilon}, \ldots, x_{n}^{\varepsilon}\right) .
$$

Proof. Since

$$
\left[x_{i}, x_{j}\right]=\frac{m}{\left(\frac{m}{x_{i}}, \frac{m}{x_{j}}\right)}=\frac{m \cdot\left[\frac{m}{x_{i}}, \frac{m}{x_{j}}\right]}{\frac{m}{x_{i}} \cdot \frac{m}{x_{j}}}=\frac{x_{i} x_{j}}{m} \cdot\left[\frac{m}{x_{i}}, \frac{m}{x_{j}}\right],
$$

it follows that

$$
\left[x_{i}, x_{j}\right]^{\varepsilon}=\frac{x_{i}^{\varepsilon} x_{j}^{\varepsilon}}{m^{\varepsilon}} \cdot\left[\frac{m}{x_{i}}, \frac{m}{x_{j}}\right]^{\varepsilon} .
$$

Therefore the result follows immediately.

Definition. Let $S=\left\{x_{1}, \ldots, x_{n}\right\}$ be a set of $n$ distinct positive integers. Let $m=\operatorname{lcm}\{S\}$. Then the reciprocal set of $S$, denoted by $m S^{-1}$, is 
defined by

$$
m S^{-1}=\left\{\frac{m}{x_{1}}, \ldots, \frac{m}{x_{n}}\right\} .
$$

Lemma 4.2. Let $S=\left\{x_{1}, \ldots, x_{n}\right\}$ be an lcm-closed set. Then the reciprocal set $m S^{-1}$ is gcd-closed.

Proof. First, for any $1 \leq i, j \leq n$, one has

$$
\left(\frac{m}{x_{i}}, \frac{m}{x_{j}}\right)=\frac{m}{\left[x_{i}, x_{j}\right]}
$$

But $S$ is lcm-closed. So there exists $1 \leq k \leq n$ such that $\left[x_{i}, x_{j}\right]=x_{k}$. Therefore

$$
\left(\frac{m}{x_{i}}, \frac{m}{x_{j}}\right)=\frac{m}{x_{k}} \in m S^{-1} .
$$

Thus the reciprocal set $m S^{-1}$ is gcd-closed.

We can give the second main result in this paper as follows.

Theorem 4.3. Let $S=\left\{x_{1}, \ldots, x_{n}\right\}$ be an lcm-closed set satisfying $\max _{x \in S}\{v(x)\} \leq 2$ and $\varepsilon$ a positive integer. Then the power LCM matrix $\left(\left[x_{i}, x_{j}\right]^{\varepsilon}\right)$ defined on $S$ is nonsingular.

Proof. This follows immediately from Lemmas 4.1 and 4.2, and Theorem 3.3 .

5. Final remarks. Let $S=\left\{x_{1}, \ldots, x_{n}\right\}$ be a set of positive integers. The set $S$ is said to be odd gcd-closed if $S$ is gcd-closed and every element in $S$ is an odd number. The set $S$ is said to be even gcd-closed if $S$ is not odd gcd-closed. By [11], we know that there is an even gcd-closed set $S$ such that the LCM matrix $\left(\left[x_{i}, x_{j}\right]\right)$ on $S$ is singular. But it is not clear if there is an odd gcd-closed set $S$ such that the LCM matrix $\left(\left[x_{i}, x_{j}\right]\right)$ on $S$ is singular. We believe that the answer to this question is negative. Furthermore, we propose the following conjecture.

Conjecture 5.1. Let $\varepsilon$ be a positive integer and let $S=\left\{x_{1}, \ldots, x_{n}\right\}$ be an odd gcd-closed set. Then the power LCM matrix $\left(\left[x_{i}, x_{j}\right]^{\varepsilon}\right)$ on $S$ is nonsingular.

The set $S$ is said to be odd lcm-closed if $S$ is lcm-closed and every element in $S$ is an odd number. The set $S$ is said to be even lcm-closed if $S$ is not odd lcm-closed. By [11], one can easily construct an even lcm-closed set $S$ such that the LCM matrix $\left(\left[x_{i}, x_{j}\right]\right)$ on $S$ is singular. We suggest another conjecture. 
Conjecture 5.2. Let $\varepsilon$ be a positive integer and let $S=\left\{x_{1}, \ldots, x_{n}\right\}$ be an odd lcm-closed set. Then the power LCM matrix $\left(\left[x_{i}, x_{j}\right]^{\varepsilon}\right)$ on $S$ is nonsingular.

By Lemmas 4.1 and 4.2, Conjecture 5.1 is equivalent to Conjecture 5.2. Namely, Conjecture 5.1 implies Conjecture 5.2, and the converse is also true.

It follows from [13] that there is an even gcd-closed set $S=\left\{x_{1}, \ldots, x_{n}\right\}$ such that the GCD matrix $\left(\left(x_{i}, x_{j}\right)\right)$ on $S$ does not divide the LCM matrix $\left(\left[x_{i}, x_{j}\right]\right)$ on $S$ in the ring $M_{n}(\mathbb{Z})$. By [13], one can also easily construct an even lcm-closed set $S$ such that the GCD matrix $\left(\left(x_{i}, x_{j}\right)\right)$ on $S$ does not divide the LCM matrix $\left(\left[x_{i}, x_{j}\right]\right)$ on $S$ in the ring $M_{n}(\mathbb{Z})$. However it is not clear if there is an odd gcd-closed (resp. lcm-closed) set $S=\left\{x_{1}, \ldots, x_{n}\right\}$ such that the GCD matrix $\left(\left(x_{i}, x_{j}\right)\right)$ on $S$ does not divide the LCM matrix $\left(\left[x_{i}, x_{j}\right]\right)$ on $S$ in the ring $M_{n}(\mathbb{Z})$. We still believe that the answer is negative. We raise the following conjectures as the conclusion of this paper.

Conjecture 5.3. Let $\varepsilon$ be a positive integer and let $S=\left\{x_{1}, \ldots, x_{n}\right\}$ be an odd gcd-closed set. Then the power GCD matrix $\left(\left(x_{i}, x_{j}\right)^{\varepsilon}\right)$ on $S$ divides the power LCM matrix $\left(\left[x_{i}, x_{j}\right]^{\varepsilon}\right)$ on $S$ in the ring $M_{n}(\mathbb{Z})$.

Conjecture 5.4. Let $\varepsilon$ be a positive integer and let $S=\left\{x_{1}, \ldots, x_{n}\right\}$ be an odd lcm-closed set. Then the power GCD matrix $\left(\left(x_{i}, x_{j}\right)^{\varepsilon}\right)$ on $S$ divides the power LCM matrix $\left(\left[x_{i}, x_{j}\right]^{\varepsilon}\right)$ on $S$ in the ring $M_{n}(\mathbb{Z})$.

Acknowledgements. The author wishes to thank Professor R. Loewy for his help and encouragement in writing this paper. The author is also grateful to the anonymous referee for valuable comments and kindly correcting grammatical errors in the first version of this paper.

\section{References}

[1] S. Beslin, Reciprocal GCD matrices and LCM matrices, Fibonacci Quart. 29 (1991), 271-274.

[2] S. Beslin and S. Ligh, Another generalization of Smith's determinant, Bull. Austral. Math. Soc. 40 (1989), 413-415.

[3] -, 一, Greatest common divisor matrices, Linear Algebra Appl. 118 (1989), 69-76.

[4] K. Bourque and S. Ligh, On GCD and LCM matrices, ibid. 174 (1992), 65-74.

[5] -, - Matrices associated with arithmetical functions, Linear and Multilinear Algebra 34 (1993), 261-267.

[6] —, - Matrices associated with classes of arithmetical functions, J. Number Theory 45 (1993), 367-376.

[7] —, - Matrices associated with classes of multiplicative functions, Linear Algebra Appl. 216 (1995), 267-275.

[8] P. Haukkanen and J. Sillanpaa, Some analogues of Smith's determinant, Linear and Multilinear Algebra 41 (1996), 233-244. 
[9] S. F. Hong, LCM matrix on an r-fold gcd-closed set, J. Sichuan Univ. Nat. Sci. Ed. 33 (1996), 650-657.

[10] - On LCM matrices on GCD-closed sets, Southeast Asian Bull. Math. 22 (1998), 381-384.

[11] - On the Bourque-Ligh conjecture of least common multiple matrices, J. Algebra 218 (1999), 216-228.

[12] -, Gcd-closed sets and determinants of matrices associated with arithmetical functions, Acta Arith. 101 (2002), 321-332.

[13] - On the factorization of LCM matrices on gcd-closed sets, Linear Algebra Appl. 345 (2002), 225-233.

[14] H. J. S. Smith, On the value of a certain arithmetical determinant, Proc. London Math. Soc. 7 (1875-1876), 208-212.

Mathematical College

Sichuan University

Chengdu 610064, P.R. China

E-mail: s-f.hong@163.net

hongsf02@yahoo.com
Department of Mathematics Technion-Israel Institute of Technology Haifa 32000, Israel

Received on 13.9.2002

and in revised form on 5.3.2003 